\title{
Laparoscopic Cholecystectomy for Acute Cholecystitis Beyond the Conventional Safety Period Proceeded by Ultrasonic Energy Feasibility and Outcome
}

\author{
Yasser M. Hatata, MD, FRCS; Nader Shaban MD; \\ Ghada Morshed MD, MRCS; and Mohamed Ebrahim, MD.
}

Department of Surgery, Fayoum University, Egypt.

Background: Timing of laparoscopic cholecystectomy for acute cholecystitis (from the onset of symptoms) is related to the degree of operative difficulty, increases substantially over time. The appropriate timing for early laparoscopic cholecystectomy in the treatment of acute cholecystitis remains controversial. However, no definitive advantages to initial conservative management and delayed laparoscopic surgery in patients suitable for surgery.

Patients \& methods: 30 laparoscopic cholecystectomies were tried for acute gallbladder disease beyond the conventional coded time of 72 hours from the onset of symptoms up to 5 weeks later. Ultra-sonic energy devices (Harmonic Scalpel and Ligasure) were the energizer tools for surgical dissection. Intra-operative difficulties \& complications, shift to other procedures, length of operations \& post-operative hospital stay and 1 month follow up for potential complications were assessed for feasibility and outcome.

Results: 28 of the 30 (93.33\%) laparoscopic cholecystectomies were successfully completed. 2 cases (6.67\%) were shifted into another procedure.

Some intra-operative difficulties in some cases were encountered in the form of variable density of omental and other structures adhesions with difficult separation (n: 28, 100\%), difficulty in grasping gallbladder fundus (n: 7, 25\%), difficult dissection at Calot triangle (n: 13, 46.43\%), cystic duct \& artery identification, isolation and clipping (n: 13, 43.33\%), difficult dissection of gallbladder off its liver bed (n: 9, 32.1\%) and difficult extraction of the gallbladder (n: 3, 10.7\%). No intra-operative complications were met. The length of operations ranged between 100-130 Min. With a mean of 115 Min. The length of post-operative stay ranged between 1-2 days with a mean of 1.5 days. Post-operative complications comprised 2 cases (7.1\%). One with biliary leakage (Clavien-Deino grading system $2 b$ ) and the other had a superficial surgical site infection at the umbilical incision (Clavien-Deino grading system1).

Conclusion: Laparoscopic cholecystectomy for acute gallbladder disease beyond the conventional coded time of 72 hours from the onset of symptoms using ultra-sonic energy devices for surgical dissection bears a high threshold of safety \& feasibility with an excellent outcome in certain patients, this is mainly evident when the operator is an experienced laparoscopic surgeon in a highly technologically equipped laparoscopic center.

Key words: Acute cholecystitis, laparoscopic cholecystectomy, ultra-sonic energy devices.

\section{Introduction:}

Cholecystitis is the most prevalent surgical condition affecting populations in industrialized countries. ${ }^{1}$ Gallstones constitute a significant health problem in developed societies, affecting $10 \%-15 \%$ of the adult population. ${ }^{2}$ Of all cholecystectomies, $10-30 \%$ is presented in the acute setting. This is most commonly $(90 \%-95 \%)$ obstructive in nature from impaction of a stone in Hartman pouch or cystic duct. ${ }^{3}$

The appropriate timing for early 
laparoscopic cholecystectomy in the treatment of acute cholecystitis remains controversial and the timing of surgery has varied in studies. ${ }^{4}$ The degree of operative difficulty increases substantially over time in acute cholecystitis and surgeons have typically used 72 hours as an arbitrary cutoff in degree of difficulty of the dissection. Generally, in the first 48 to 72 hours of symptoms the tissue planes are edematous but structures are identifiable and the tissue planes separate without much difficulty. After 72 hours, the tissues become more friable and separate less well, the important structures are less likely to be seen well, and there is often more obscurative bleeding. For this reason, it is important to consider operating early in acute cholecystitis if the patient does not respond to conservative measures and antibiotic therapy after 24 hours of observation. ${ }^{5}$

Electro-cautery remains the main energy form used during laparoscopic dissection. However, because of its documented risks, especially those related to visceral injury, search for alternative forms of energy that can be used in laparoscopic dissection and even coagulating and sealing vessels and ducts began very early during the evolution of laparoscopic cholecystectomy itself. Ultrasonic energy dissection was reported using different devices and terminology [Harmonic scalpel, ultrasonic shears, ultrasonically activated coagulating shears (UACS), Ultracision Harmonic Shears (UHS), Cavitron Ultrasonic Surgical Aspirator (CUSA)and [Liga-Sure]. ${ }^{6}$ The Harmonic Scalpel is the leading ultrasonic cutting and coagulating surgical device, offering surgeons important benefits including: Minimal lateral thermal tissue damage, minimal charring and desiccation. Harmonic Scalpel technology reduces the need for ligatures with simultaneous cutting and coagulation; moreover there is not electricity to or through the patient. ${ }^{7}$ Harmonic Scalpel has a greater precision near vital structures and it produces minimal smoke with improved visibility in the surgical field. In retrospective series laparoscopic cholecystectomy performed with Harmonic Scalpel was demonstrated feasible and effective with minimal operating time and blood loss; it was reported also a low conversion rate. ${ }^{8}$

\section{Patient and methods:}

It is a prospective study that was conducted in the Department of Surgery, Faculty of Medicine, Fayoum University from the period of August 2013 till October 2014. A total of 30 patients of acute cholecystitis, 72 hours from beginning of the symptoms up to 5 weeks later were included in the study. Of these 30 patients, 5 cases were accidently diagnosed intra-operatively as having a recent acute attack during elective laparoscopic cholecystectomy for a chronic gallbladder stone disease, accurate retrospective analysis of their history demonstrated the appearance of acute symptoms more than 72 hours of the operation. The rest were pre-operatively diagnosed as acute cholecystitis .

Acute cholecystitis was established based upon the Revised Tokyo Guidelines Diagnostic Criteria for Acute Cholecystitis , as follows:-

A. Local signs of inflammation, etc.: (1) Murphy's sign, (2) right upper quadrant mass/ pain/tenderness.

B. Systemic signs of inflammation, etc.: (1) Fever, (2) elevated C-Reactive Protein, (3) abnormal White Blood Cell count.

C. Imaging findings: Imaging findings characteristic of acute cholecystitis, basically:-abdominal ultra-sonography; which was enough for imaging diagnosis.

Radiology ultra-sonography results were defined as positive for acute cholecystitis if they demonstrated cholelithiasis (in the calculous variety) plus any one of the following secondary findings: Wall thickening greater than $3 \mathrm{~mm}$, pericholecystic fluid, a sonographic Murphy's sign,a striated gallbladder wall, mucosal sloughing, intramural gas, sludge within the gallbladder and gallbladder dilatation of greater than 5 $\mathrm{cm}$ in transverse diameter.

Definite Diagnosis :

(1) One item in A + one item in B were positive. 


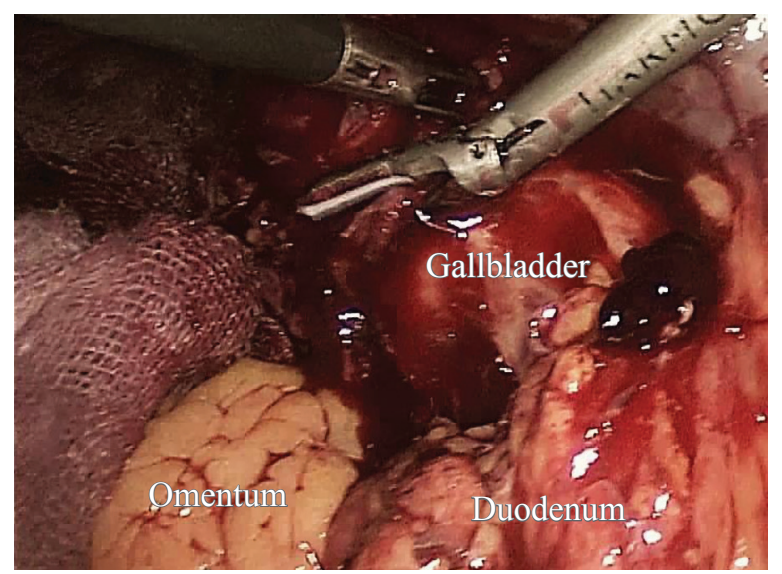

Figure (1): An intra-operative picture showing severely inflamed gallbladder with omental \& duodenal adhesions.

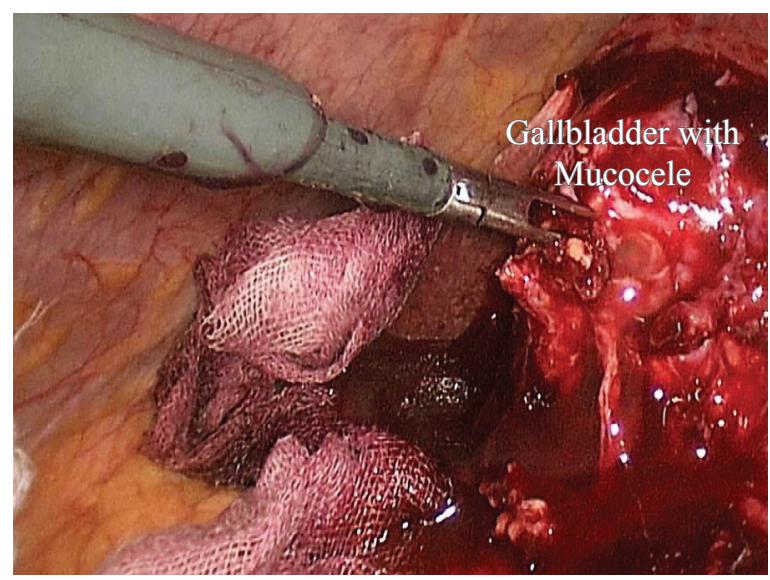

Figure (3): An intra-operative picture showing a distended gallbladder with mucocele.

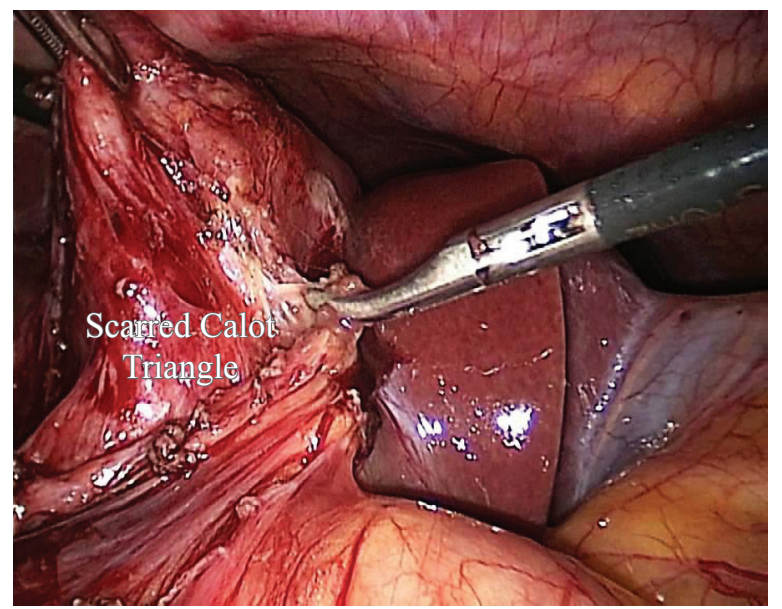

Figure (5): An intra-operative picture showing dissection at scarred Calot triangle.

(2) C confirms the diagnosis when acute cholecystitis was suspected clinically.

Other acute abdominal diseases were

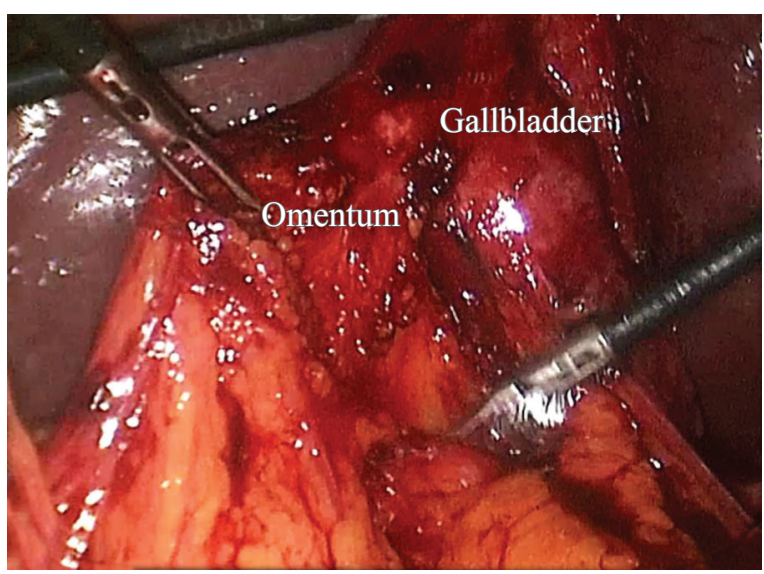

Figure (2): An intra-operative picture showing severely inflamed gallbladder with omental\& stomach adhesions.

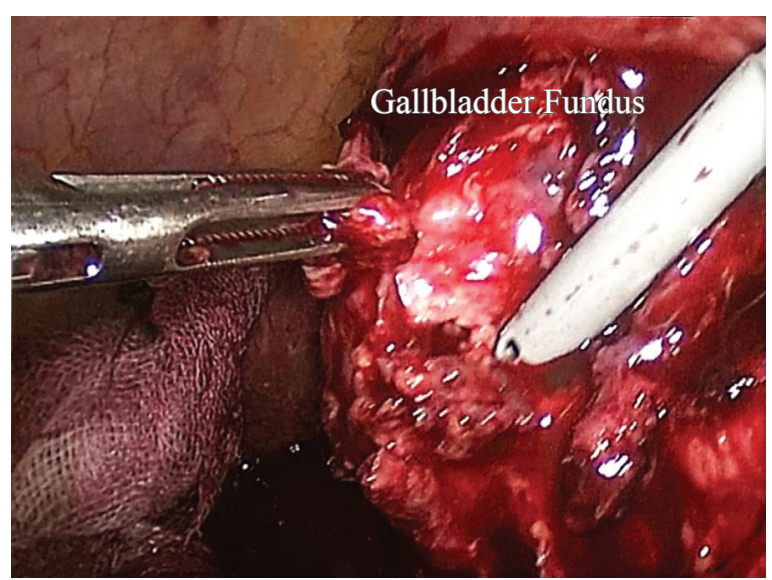

Figure (4): An intra-operative picture showing a hole made in the fundus of a distended gallbladder aiming at suction of its contents for emptying.

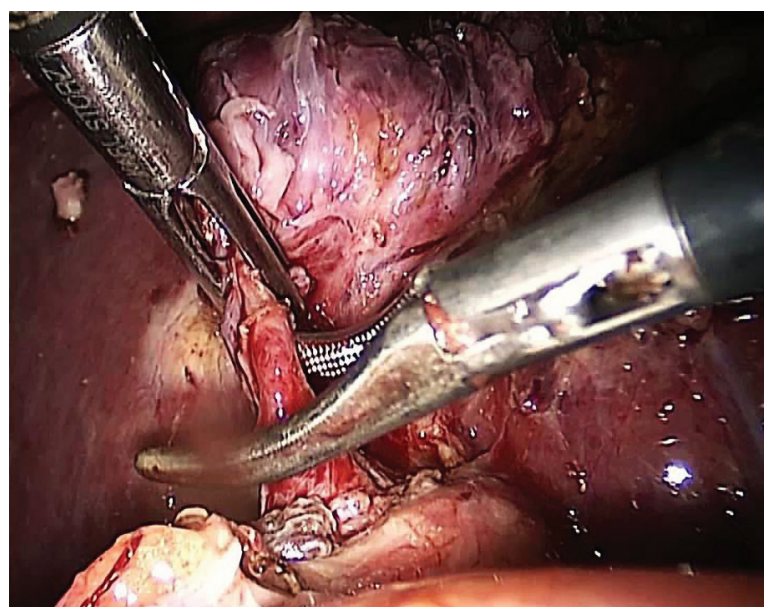

Figure (6): An intra-operative picture showing skeletonized cystic duct.

excluded through the routine laboratory and imaging work up, as appropriate for every case. 


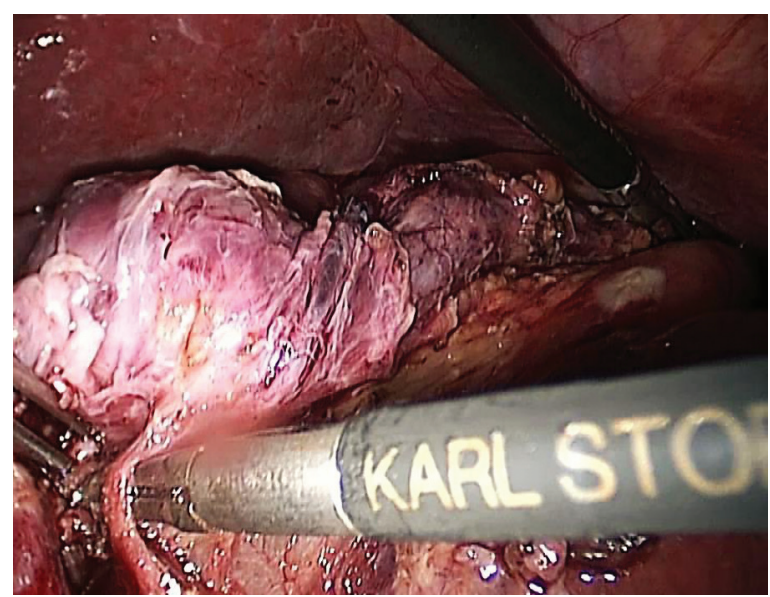

Figure (7): An intra-operative picture showing skeletonized cystic artery.

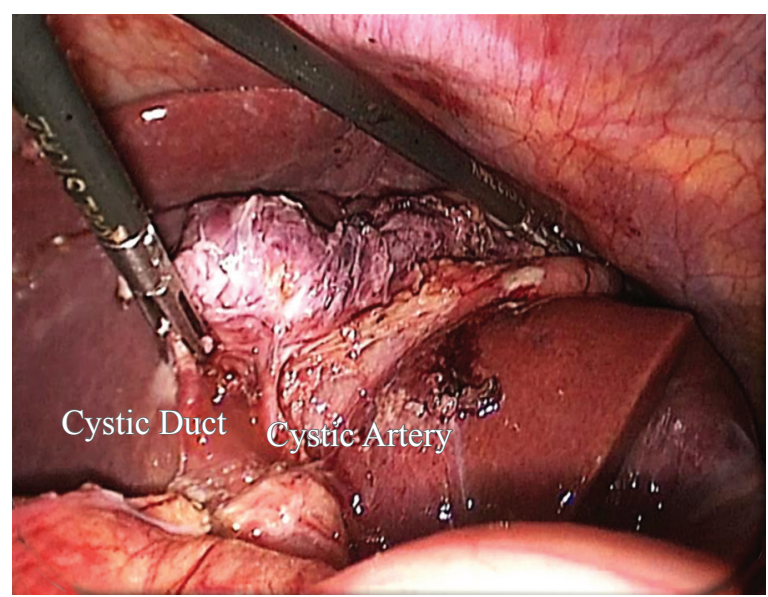

Figure (8): An intra-operative picture showing Critical View of Safety.

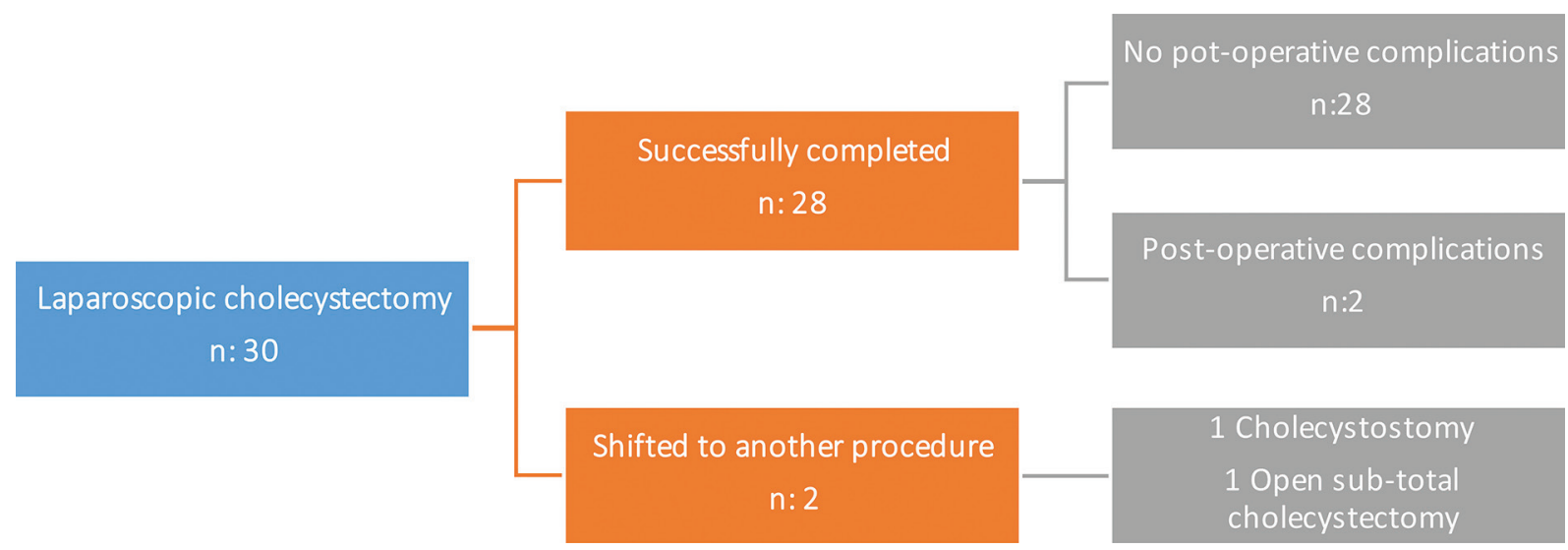

Figure (9): Flow chart of study.

We excluded patients above 60 years age, choledocholithiasis with abdominal ultrasonography, having co-morbid conditions out of ASA I \& II or its acute cholecystitis was secondary to a systematic illness, pregnancy/ history of missed periods in premenopausal females, Jaundice, cholangitis, and those who refused surgery.

A written informed consent was taken from all patients. Ethical approval was obtained from the ethical scientific committee of the Faculty of Medicine, Fayoum University.

Pre-operatively all admitted patients were re-evaluated by a detailed history, thorough examination and revision of their investigations. Patients with a present acute attack were kept on nil per oral, intra-venous fluids, parentral antibiotics and parentral analgesia; as appropriate.
Statistical analysis:

Categorical variables were quoted as the number and percentage. Quantitative variables were summarized as the range and mean.

Surgical technique:

Laparoscopic cholecystectomy was done under general anesthesia with muscle relaxants and endo-tracheal intubation. Patients were put supine on the operating table with routine application of crepe bandages as a mechanical anti-thrombotic maneuver. Routine prophylactic antibiotic (one of the $3^{\text {th }}$ generation cephalosporins) was administered with induction of anesthesia after performing the sensitivity test at ward. Array of the operators and equipments entailed that, the surgeon stood to the left of the patient. The first assistant stood to the patient's right. A laparoscopic video camera operator stands 
to the left side of the surgeon. The video monitor was placed on the patient's right at the level of his shoulder. Insufflation was commenced by an open technique. Standard four port technique was used for the laparoscopic cholecystectomy. Ultra-sonic energy devices were the tools of dissection, principally HARMONIC SCALPEL ${ }^{\circledR}$ (Ethicon Endo-Surgery of Johnson \& Johnson, Inc., Cincinnati, Ohio, USA) and

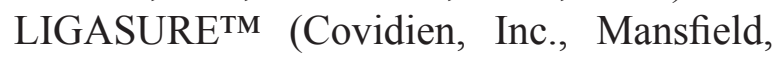
Massachusetts, USA). Conventional technique for laparoscopic cholecystectomy was proceeded without the use of either intra-operative cholangiography or ultrasonography. Intra-operative modifications were adopted according to operative circumstances and surgeon's preference eg. Gallbladder decompression. An abdominal drain was left at the end of surgery.

Operative difficulties were determined as difficulties in/with:

- Dense omental \& other structure adhesions to gallbladder and their dissection away,

- Dissection of hepato-cystic triangle with identification \& isolation of both cystic duct \& artery,

- Clipping of wide mouthed cystic duct,

- Dissection of the gallbladder from liver bed, and

- $\quad$ Extraction of the gallbladder; if any.

- Intra-operative complications were determined as occurrence of:

- Significant hemorrhage,

- Major source bile leakage,

- Bile duct injury, and

- Nearby organs injury; if any.

Conversion to laparotomy or shift to cholecystostomy was recorded. The length of the operations (in minutes, starting from umbilical incision) was recorded.

Post-operatively:-All patients were permitted to receive oral fluids and encouraged to be ambulated as tolerated. The drain was removed when the output quantity fell below $50 \mathrm{ml}$. Per a day except cases of biliary leakage. None of the selected patients was specifically managed in a day case setting or with specific anaesthesia or pain control.
Length of post-operative hospital stay was recorded in days from surgery.

Post-operative complications both early and late: Up to 1 month of the surgery, were recorded. These were in the form of:-hemorrhage, hematoma, surgical site infection, port-site herniae, jaundice, bile leakage, bile duct injury and bile duct stricture; if any. They were rated according to the Clavien-Deino grading system .

\section{Results:}

At the time of study, 30 instances underwent a trial of laparoscopic cholecystectomy, more than 72 hours from the onset of symptoms up to 5 weeks later. Ultra-sonic energy devices (Harmonic Scalpel and LigaSure) were the energizer tools for surgical dissection.

Our patients (n: 8, 26.67\%) were males while (n: 22, 73.33\%) were females. Their ages ranged from 19 years to 59 years, with a mean of 39 years. Patients of ASA 1 were (n: $23,67.67 \%$ ); while of ASA 2 were (n: 7, $23.33 \%$ ).

The indications of surgery were acute calculouscholecystitis (n: 25, 83.33\%) and chronic calculouscholecystitis, diagnosed intra-operatively as had a recent attack of acute cholecystitis, (n: 5, 16.67\%).

The mean interval period from the onset of symptoms till surgery was 3 weeks.

Of the 30 laparoscopic cholecystectomies, $28(93.33 \%)$ were successfully completed ending with complete removal of the gallbladder. 2 cases $(6.67 \%)$ shifted into another procedure. One was converted to laparotomy because of failure of precised identification and proper separation of the structures forming the gallbladder phlegmon ending by sub-total cholecystectomy. The other one was proceeded to cholecystostomy because of heavily scarred Calot triangle making its dissection a matter of danger both laparoscopically and open.

- Intra-operative difficulties: These were encountered in the successfully completely group (n: $28,93.33 \%$ ) were in the form of:

- Variable density of omental and other structures adhesions with difficult separation 
(n: 28, 100\%).

- Difficulty in grasping gallbladder fundus (because of wall oedema or distended gallbladders) necessitated their empting (n: 7, $25 \%$ ).

- Difficult dissection at Calot triangle (n: 13, 46.43\%).

- Cystic duct \& artery identification, isolation and clipping (n: 13, 43.33\%).

- Difficult dissection of gallbladder off its liver bed (n: 9, 32.1\%).

- Difficult extraction of the gallbladder necessitated surgical site extension (n: 3, $10.7 \%)$.

The length of operation was (100-130Min) with a mean of 115 Min. Post-operative hospital stay was (1-2 days) with a mean of 1.5 days.

All abdominal drains were removed 24 hours after the operation except one stayed for 1 week. No intra-operative complications were met.

Post-operative complications comprised (n: 2 cases, $7.1 \%$ ). One with biliary leakage (Clavien-Deino grading system $2 \mathrm{~b}$ ) treated on a conservative basis, after prompt exclusion of major duct injury, by keeping the abdominal drain for 1 week until the bile efflux spontaneously ceased. The other case had a superficial surgical site infection at the umbilical incision (Clavien-Deino grading system1) which was the exit of the inflamed gallbladder, also needed just a conservative management.

\section{Discussion:}

Cholecystitis is the most prevalent surgical condition affecting populations in industrialized countries. ${ }^{1}$ Acute cholecystitis hadinitiallybeen consideredacontraindication to laparoscopic cholecystectomy because of the higher incidence of complications than in non-acute cholecystitis. ${ }^{9}$ The appropriate timing for early laparoscopic cholecystectomy in the treatment of acute cholecystitis remains controversial and the timing of surgery has varied in studies. ${ }^{10}$ Many studies tried different operative timing from the onset of the acute attack. Results showed that, delay of laparoscopic cholecystectomy beyond
72 hours from the onset of the acute attack neither increases operative difficulty nor prolong recovery with no clinical relevant effect on conversion rates, operative times, morbidity, and postoperative hospital stay. It might be more cost effective. ${ }^{11,12,13}$

In our study, laparoscopic cholecystectomy for acute gallbladder disease was performed more than 72 hours from the onset of symptoms up to 5 weeks later.

To our knowledge, no study determined intra-operativedifficulties duringlaparoscopic cholecystectomy in its acute pattern. We assume these in the settings of:-variable density of omental and other structures adhesions, difficulty in grasping gallbladder fundus, difficult dissection at Calot triangle, cystic duct \& artery: Identification-isolationclipping, difficult dissection of gallbladder off its liver bed and difficult extraction of the gallbladder. All difficulties in the completed group were been overcome with no impact upon any intra-operative morbidity.

In our study no significant intra-operative complications were met specially bile duct injury compared to other study Bile duct injury was $0.6 \% .^{14}$

Conversion to another procedure was $6.67 \%$. Compared to other studies which ranges from5.6 to $32 \% .{ }^{15}$

The mean interval period from the onset of symptoms till surgery was 3 weeks. This was not found to have any significant bearing on completion / conversion of the procedure. And was parallel to Wang et al. Who showed that no impact for the timing of laparoscopic cholecystectomy on conversion rate. ${ }^{16}$

Electro-cautery remains the main energy form used during laparoscopic dissection, however because of its documented risks,alternative forms of energy that can be used in laparoscopic dissection and even coagulating and sealing vessels and ducts began very early during the evolution of laparoscopic cholecystectomy itself. Ultrasonic energy dissection was reported using different devices and terminology [Harmonic scalpel, ultrasonic shears, ultrasonically activated coagulating shears (UACS), Ultracision Harmonic Shears (UHS), 
Cavitron Ultrasonic Surgical Aspirator (CUSA)and Liga-Sure]. ${ }^{17}$

The length of operations ranged between 100-130 Min. With a mean of 115 Min. Compared to other study which was with a mean 50 Min. ${ }^{18}$

The length of post-operative stay ranged between 1-2 days with a mean of 1.5 days compared to other study which was with a mean 2.7 days. ${ }^{18}$

In our study we used Harmonic scalpel and Liga-sure with a high threshold of safety $\&$ feasibility with an excellent outcome in our patients.

\section{Conclusion:}

Laparoscopic cholecystectomy for acute gallbladder disease, beyond the conventional coded time of 72 hours from the onset of symptoms, using ultra-sonic energy devices for surgical dissection; bears a high threshold of safety \& feasibility with an excellent outcome.

\section{Reference:}

1- Elwood DR: Surgical Clinics of North America, Biliary Tract Surgery. Cholecystitis In: J.L. Munson (Ed.) 2008; 88(6) 1241-1252.

2- Stinton LM, Shaffer EA: Epidemiology of Gallbladder Disease: Cholelithiasis and Cancer In: Gut and Liver 2012; 6(2): 172-187.

3- Cuschieri A: Disorders of the Biliary Tract In: Sir Alferd Chushieri, Robert J.C. Steel and Abdool Rahim Moossa (Eds.): Essential Surgical Practice, 4th ed., 2002 Arnold, 375-452.

4- Siddiqui T, MacDonald A, Chong PS, Jenkins JT: Early versus delayed laparoscopic cholecystectomy for acute cholecystitis: A meta analysis of randomized clinical trials. The American Journal of Surgery 2008; 195(1): 40-47.

5- Litwin DEM, Cahan MA: Laparoscopic Cholecystectomy, Surgical Clinics of North America. Biliary Tract Surgery 2008; 88(6), 1295-1313.

6- Sasi W: Dissection by Ultrasonic Energy Versus Monopolar Electrosurgical Energy in Laparoscopic Cholecystectomy. JSLS 2010; 14(1): 23-34 . [PubMed: 20412640]

7- Catena F, Ansaloni L, Saverio SD, Gazzotti F, Coccolini F, Pinna AD: The HAC Trial
(Harmonic for Acute Cholecystitis) Study. Randomized, double-blind, controlled trial of Harmonic $(\mathrm{H})$ versus Monopolar Diathermy (M) for laparoscopic cholecystectomy (LC) for acute cholecystitis (AC) in adults. Trials 2009; 10: 34. Http://www.ncbi.nlm.nih.gov [PubMed: 2693511]

8- Hüscher CG, Lirici MM, Di Paola M, Crafa F, Napolitano C, Mereu A, Recher A, Corradi A, Amini M: Laparoscopic cholecystectomy by ultrasonic dissection without cystic duct and artery ligature. Surg Endosc 2003; 17(3): 442-451. [PubMed: 12399846]

9- Cushieri A, Dubois F, Mouiel J, Mouiel P, Becker H, Buess G, Trede M and Troidl H: The European experience with laparoscopic cholecystectomy. Am J Surg 1991; (161) 385-387. [PubMed: 1825763]

10- Siddiqui T, MacDonald A, Chong PS, Jenkins JT: Early versus delayed laparoscopic cholecystectomy for acute cholecystitis: A meta analysis of randomized clinical trials. The American Journal of Surgery 2008; 195(1): 40-47.

11- Low JK, Barrow P, Owera A, Ammori BJ: Timing of laparoscopic cholecystectomy for acute cholecystitis: Evidence to support a proposal for an early interval surgery. Am Surg 2007; 73(11) 1188-1192. [PubMed:18092660]

12- Popkharitov AI: Laparoscopic cholecystectomy for acute cholecystitis. Langenbecks Arch Surg 2008; 393(6): 935 941. [PubMed: 18299882]

13- Lee AY, Carter JJ, Hochberg MS, Stone AM, Cohen SL, Pachter HL: The timing of surgery for cholecystitis: A review of 202 consecutive patients at a large municipal hospital. Am J Surg 2008; 195(4): 467-470. [PubMed18361924]

14- Mahatharadol V: Bile duct injuries during laparoscopic cholecystectomy: An audit of 1522 cases. Hepato-gastroenterology 2004; $51,12-14$.

15- Kanakala V, Borowski DW, Pellen MGC, Dronamraju SS, Woodcock SAA, Seymour K, Attwood SEA, Horgan LF: Risk factors in laparoscopic cholecystectomy: A multivariate analysis. International Journal of Surgery 2011; 9(4): 318-323

16- Wang YC, YangHR, Chung PK, Jeng LB, Chen RJ: Urgent Laparoscopic Cholecystectomy in the Management of Acute Cholecystitis: Timing does not Influence Conversion Rate. Surg Endosc 2006; 20: 806-808.

17- Sasi W: Dissection by Ultrasonic Energy 
Versus Monopolar Electrosurgical Energy in Laparoscopic Cholecystectomy. JSLS 2010; 14(1): 23-34. [PubMed: 20412640]

18- Davila D, Manzanares C, Picho ML, Albors
P, Cardenas F, Fuster E, et al: Experience in the treatment (early vs. Delayed) of acute cholecystitis via laparoscopy. Cirugia Espanola 1999; 66(1): 233. 